\title{
Unchaining people with mental disorders: medication is not the solution-ERRATUM
}

Vikram Patel and Kamaldeep Bhui

https://doi.org/10.1192/bjp.2017.3,

Published by Cambridge University Press, 4 January 2018.

In the original publication of this editorial, the affiliation for Kamaldeep Bhui was stated incorrectly. The correct affiliation is:

Cultural Psychiatry \& Epidemiology, The Centre for Psychiatry, Wolfson Institute of Preventive Medicine, Barts and The London School of Medicine \& Dentistry, Queen Mary University of London, London, UK and East London NHS Foundation Trust, London, UK.
This change has been made to the original article online. The publisher apologises for this error.

\section{Reference}

Patel V, Bhui K. Unchaining people with mental disorders: Medication is not the solution. The British Journal of Psychiatry 2018; 212(1), 6-8. doi:10.1192/ bjp.2017.3 